\title{
Electrochemical Polymerization and Characterization of Polyaniline/Carbon Nanotube Composite Films
}

\author{
Liang $\mathrm{ZHAO}^{1, \mathrm{a}}$, Xiao-Xia LI ${ }^{1,2, \mathrm{~b},{ }^{*}}$, Yu-Xiang GUO ${ }^{1,2, \mathrm{c}}$, De-Yue MA ${ }^{1, \mathrm{~d}}$, \\ Zhang-Bin HUANG ${ }^{1, \mathrm{e}}$ \\ ${ }^{1}$ State Key Laboratory of Pulsed Power Laser Technology, Hefei 230037, China \\ ${ }^{2}$ Anhui Key Laboratory of Infrared and Low Temperature Plasma, Hefei 230037, China \\ akobepya@163.com, ${ }^{\mathrm{b}}$ Ixxhong@163.com, ${ }^{\mathrm{C}} 31355126 @ Q Q . c o m,{ }^{\mathrm{d}}$ madeyue@163.com, \\ 4408867079@QQ.com \\ ${ }^{*}$ Corresponding author
}

Keywords: Polyaniline, Carbon Nanotubes, Cyclic Voltammetry, Electrochromic.

\begin{abstract}
Polyaniline/Carbon Nanotube (PANI/CNT) composite films doped with dodecyl benzene sulfonic acid were synthesized by cyclic voltammetry on an ITO-deposited polyethylene terephthalate substrate. The SEM and FTIR were used to characterize the micro-morphology and chemical structure. The effect of CNT concentration on the electrical conductivity of the film was measured by a four-probe resistivity instrument. The electrochromic behavior of the composite film was characterized by a spectral photometer and an electrochemistry analyzer. The results show that CNTs act as a skeleton on which aniline can polymerize in the polymerization of PAN/CNT composite film. The film exhibits a porous network structure which can offer more approaches for electron transport and facilitate ion transmission. The cyclic voltammetry current density in the third polymerization cycle is the maximum because of the dense criss-cross network in the film. The CNT concentration takes great effects on the performance of PANI/CNT composite film, and the electric conductivity of the film improves with the CNT concentration rising within some limits. The CNT percolation threshold of the film is about $3.5 \mathrm{wt} . \%$. The PANI/CNT film polymerized at a CNT concentration of $2.5 \mathrm{wt} . \%$, shows a reflectance change range of $38.8 \%$ at $540 \mathrm{~nm}$ as well as a coloration efficiency of $386.4 \mathrm{~cm}^{-2} / \mathrm{C}$, which means that the PANI/CNT composite film has good behavior of voltage response.
\end{abstract}

\section{Introduction}

Polyaniline (PANI) is a kind of conductive polymer which has benzene ring of conjugate long-chain molecules. It is actively investigated and employed due to the cheapness and easy availability of raw materials, ease of synthesis, good environmental stability, high electrical conductivity and simple doping/dedoping chemistry ${ }^{[1]}$. One of the more popular and attractive property is electrochromism and the ease of reversible color-conversion, which may be widely used in electrochromic devices (ECDs $)^{[2,3,4]}$. However, the long-term application of PANI-based ECD has been restricted due to the poor ion transport behavior and short cycle life of PANI.

Carbon nanotubes (CNTs) have unique mechanical, electrical, magnetic, optical and thermal properties and are being studied for some special applications, such as molecular electronics and sensors etc. ${ }^{[5,6]}$. They have been good candidates for complementing the weak electric conductivity of some materials since discovered, because of their high electrical conductivity, strength, stability and large aspect ratio.

Previous research has proved that the polymerization of aniline in the presence of CNTs produces a coaxial coating of the CNTs with PANI ${ }^{[7]}$. One of the important characteristics of PANI/CNTs is an interaction between the $\pi$-bonded surfaces of CNTs with conjugated structure of PANI. This 
interaction of CNTs, as an electron acceptor, and PANI, as an electron donor, facilitates charge transfer between these two materials and stabilizes the delocalized electronic structures. The PANI/CNT composites introduce the excellent conducting and nanostructure properties of CNTs into PANI polymer matrix. The synergistic effects of components promote the conductivity, reversibility of PANI and the dispersity of $\mathrm{CNTs}^{[8]}$. Zuzana et al. used in-situ polymerization to enhance thermal stability of MWCNTs/PANI composites ${ }^{[9]}$; Zhou et al. prepared PANI/CNTs composites with core-shell structure consisting of polyaniline and carbon nanotubes as supercapacitor electrode materials via in-situ polymerization method ${ }^{[10]}$. Marcela et al. synthesized nanostructures of PANI with embedded carbon nanotubes through a chemical method of self-organization and evaluated the specific capacitance. The results confirmed that the developed morphologies brought on by CNTs strongly influenced the property of PANI ${ }^{[11]}$.

Over the past several years, the preparation of PANI/CNT composite is mainly accomplished by chemical polymerization, which leads to a powder-like composite product ${ }^{[12]}$. The humdrum form of the product will restrict the exploration and application of PANI/CNT composites in some special fields. In this paper, we prepared PANI/CNT composite film doped with dodecyl benzene sulfonic acid (DBSA) by electrochemical polymerization. The composite film adheres to the electrode compactly and uniformly, and can show a good and stable electrochromic behavior.

\section{Experimental}

\section{Raw Materials}

Aniline with a purity of $99 \%$ was distilled twice and then used as the monomer of PANI. Multi-walled carbon nanotubes (with an external diameter of 20 40nm and a length=of 1 30 $\mu \mathrm{m}$ ) were obtained by ethylene chemical vapor deposition(CVD) using $\mathrm{Al}_{2} \mathrm{O}_{3}$ supported $\mathrm{Fe}_{2} \mathrm{O}_{3}$ catalysts $^{[13]}$, and were acidized and dispersed for 2 hours in a baker. The other reagents, such as dodecyl benzene sulfonic acid (DBSA) and ethyl alcohol $\left(\mathrm{C}_{2} \mathrm{H}_{5} \mathrm{OH}\right)$ are all analytically pure.

\section{Electro-polymerization}

The electrochemical polymerization of aniline in the presence of CNTs was performed in the CHI $760 \mathrm{D}$ electrochemistry workstation, where potential zone was set from -0.2 to $1.0 \mathrm{~V}$, and scan rate was at $56 \mathrm{mV} / \mathrm{s}$. Indium tin oxide (ITO) film $(\mathrm{Rsh}=8 \Omega / \mathrm{sq})$ deposited on a polyethylene terephthalate (PET) substrate was used as the working electrode. The ITO/PET composite substrate was treated by the following procedures including ultrasonic cleaning in absolute ethyl alcohol for 15min, and then in deionized water for $15 \mathrm{~min}$. A Pt sheet was served as the counter electrode. The reference electrode was saturated calomel electrode.

The electrochemical bath solution was composed of $0.25 \mathrm{~mol} \cdot \mathrm{L}^{-1}$ aniline monomer, $0.6 \mathrm{~mol} \cdot \mathrm{L}^{-1}$ DBSA, $5 \mathrm{~mol} \cdot \mathrm{L}^{-1} \mathrm{C}_{2} \mathrm{H}_{5} \mathrm{OH}$ and CNTs with a series of concentration dispersed in deionized water. The CNT concentration was 0 wt. $\%, 0.5$ wt. $\%, 1.5$ wt. $\%, 2.5$ wt. $\%, 3.5$ wt. $\%, 4.5$ wt. $\%$ and 5.5 wt. $\%$, and the corresponding products were called P-0, CP-1, CP-2, CP-3, CP-4, CP-5, and CP-6, respectively.

\section{Characterization Techniques}

The morphologies of PANI/CNT composite films were characterized in a JEOL JSM-6700F scanning electron microscopy (SEM). The infrared spectra of the composite film was carried out with a FTIR VER TEX 80+HYPERION 2000 spectrometer with a swept area between 400 and $2500 \mathrm{~cm}^{-1}$; Electrochemical cyclic voltammetry $(\mathrm{CV})$ curves were obtained in a CHI 760D electrochemistry workstation; The electrical conductivity was measured by an SX-1934 four-point probe resistivity instrument at the room temperature; The reflectance spectra of the film were obtained in a HITACHI U-4100 UV/vis/NIR spectrometer at 400 800nm range. 


\section{Results and Discussion}

\section{Morphologies}

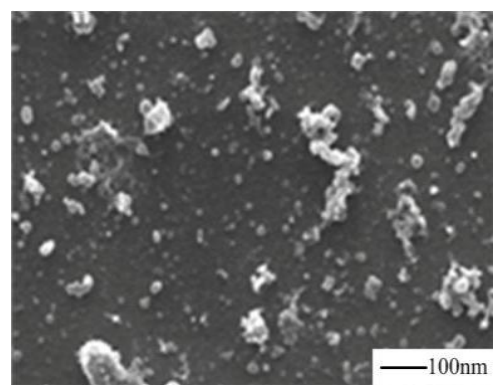

(a)

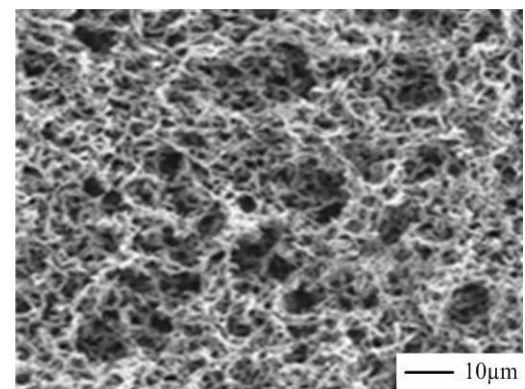

(b)

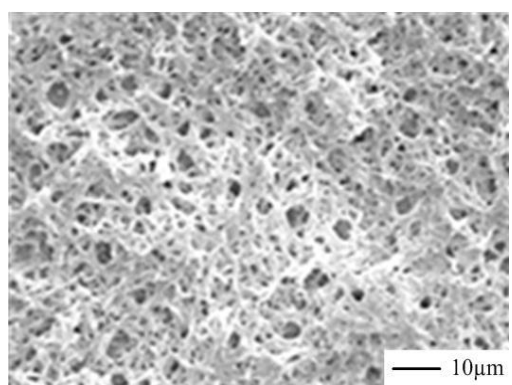

(c)

Fig.1 The SEM Images of PANI/CNT Composites Electrochemically Polymerized after (a) 1cycle (b) 2 cycles and (c) 3 cycles

The morphology images of polymer composite films prepared by cyclic voltammetry with one cycle, two cycles and three cycles, are shown in Fig.1, respectively. It can be clearly seen that the film exhibits the early time of polymerization phenomena after one cycle, and there are some clustered nucleus on the ITO/PET substrate. These clustered nucleuses may be oligomer of PANI. However, the film has a loose network structure after two cycles, which is composed of criss-cross PANI/CNTs composite fibers. During the second cyclic voltammetry cycle, polyaniline fibers grow radially and quickly around the CNTs, and form a loose three-dimensional network structure. After three polymerization cycles, the film exhibits a denser network, which is obviously different from the former. The cage-like voids among the composite fibers become shrunk and disappear. CNTs play a role of framework in the synthetic process of PANI/CNT films. According to the SEM images, it is found that the work electrode is completely covered by PANI/CNT composites after three cycles. The porous network structure of PANI/CNT films can offer more approaches for electron transport and facilitate ion transmission ${ }^{[14]}$.

\section{Electropolymerization CV Curves of PANI/CNTs}

The typical CV curves of PANI/CNT polymerization process are showed in Fig 2. The CV curves has two pairs of distinct redox current peaks centered roughly at $0.2 \mathrm{~V}$ and $0.6 \mathrm{~V}$, and their peak heights increase with the cycle index increasing in the first three periods. The peak height has reduced slightly when the forth cycle finished. From the cycle one and two curves, in other words, the "Phase ring" can be found, which proves that crystal nucleus have formed on the electrode surface according to the electro-crystallization theory ${ }^{[15]}$. The $\mathrm{CV}$ current density of the third polymerization cycle is the maximum because of the dense criss-cross network in the film. The "Phase ring" in the CV curve disappears as the polymerization continues, and the current density reduces, too. In conclusion, the PANI/CNTs composite film can cover all the surface of the electrode in three cycle period with a good and even 3D net structure ${ }^{[16]}$.

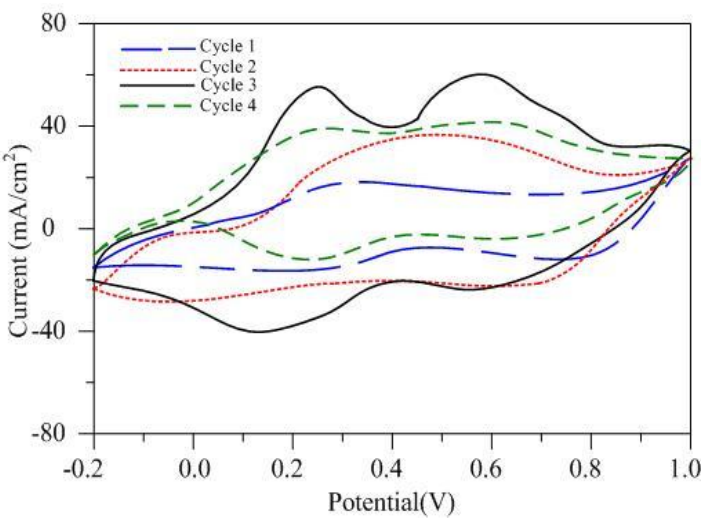

Fig.2 The CV Curves of PANI/CNTs Polymerization Process 


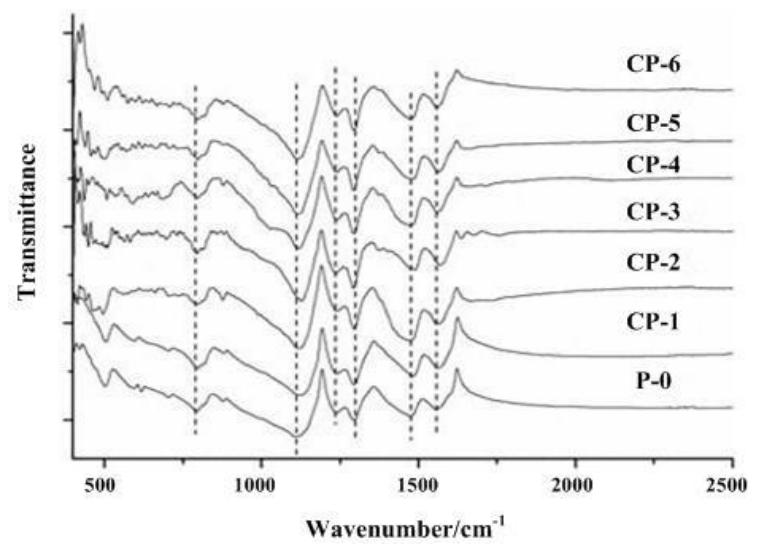

Fig.3 FTIR Patterns of PANI/CNTs with Various CNTs Content

\section{FTIR Spectra of PANI/CNTs}

The FTIR spectra of the pure PANI and PANI/CNT composites with a series of CNT concentration are recorded in the region $500 \sim 2500 \mathrm{~cm}^{-1}$, and the patterns are showed in Fig. 3. The absorption bands at $1555 \mathrm{~cm}^{-1}, 1470 \mathrm{~cm}^{-1}$ and $1238 \mathrm{~cm}^{-1}$ were attributed to the vibration of aromatic ring skeleton, $\mathrm{N}-\mathrm{B}-\mathrm{N}$ and $\mathrm{C}-\mathrm{H}$ among benzene rings, respectively ${ }^{[17,18,19]}$. The weak absorption peak at $790 \mathrm{~cm}^{-1}$ is attributed to the bending vibration of $\mathrm{C}-\mathrm{H}$ in aromatic ring skeleton ${ }^{[20]}$. The strongest band appearing near $1108 \mathrm{~cm}^{-1}$ is called "Electronic state peak", which can be used to represent the electric conductivity of PANI The sharper and higher the peak at $1108 \mathrm{~cm}^{-1}$, the better electric conductivity of $\mathrm{PANI}^{[21]}$. The electronic state peaks at $1108 \mathrm{~cm}^{-1}$ in the FTIR patterns of PANI/CNTs are stronger than that of pure PANI, and become stronger and stronger with the concentration of CNTs increasing. The results indicate that the CNTs have involved in the polymerization of PANI, and the synergistic effect between CNTs and PANI has established a conductive network which can increase the effective charge delocalization and electric conductivity ${ }^{[2]}$.

\section{Reflectance Spectra and Coloration Efficiency}

Due to the superior electrical conductivity, The CP-4 composite film is chosen for the further study of its electrochromic property. The reflectance spectra of the CP-4 films are showed in Fig.4. It can be seen that the largest difference of reflectivity peak value occurs near $540 \mathrm{~nm}$, and the largest reflectance change range of $38.8 \%$ can be gotten when the applied potential shifts from $-0.4 \mathrm{~V}$ up to $0.8 \mathrm{~V}$ with an increasing step of $0.2 \mathrm{~V}$. The PANI/CNT film shows steadily reversible electrochromic characteristics. The relationship between the optical density change $(\Delta \mathrm{OD})$ at $540 \mathrm{~nm}$ and the charge density are shown in Fig.5, respectively. The coloration efficiency, which is defined as the slope of the plot, is $368.4 \mathrm{~cm}^{-2} \mathrm{C}^{-1}$. The electrochromic behavior of the PANI/CNT film is better than that of a typical PANI film ${ }^{[24]}$.

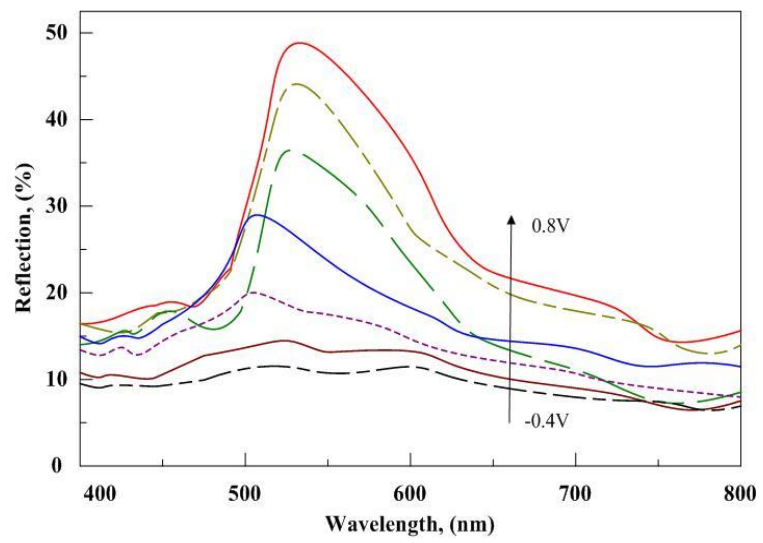

Fig.4 The Reflectance Spectra of PANI/CNTs Film between -0.4 and $0.8 \mathrm{~V}$ 


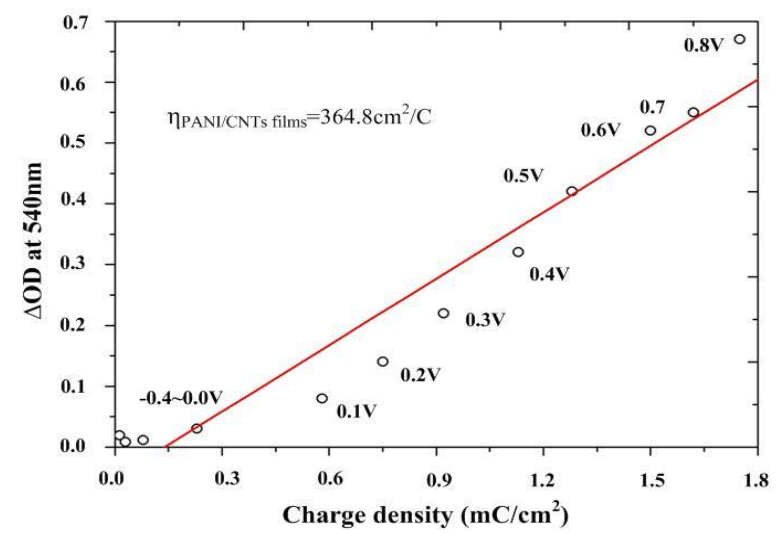

Fig.5 The Relationship between the Optical Density Change ( $\Delta O D)$ at 540nm and the Charge Density

\section{Electrical Conductivity}

Tab.1 Room Temperture Electric Conductivities of PANI/CNTs with Different CNTs Concentration

\begin{tabular}{ccc}
\hline PANI/CNTs & CNT concentration (wt.\%) & Conductivity $\left(\mathrm{S} \cdot \mathrm{cm}^{-1}\right)$ \\
\hline P-0 & 0 & 1.123 \\
CP-1 & 0.5 & 1.927 \\
CP-2 & 1.5 & 9.224 \\
CP-3 & 2.5 & 19.465 \\
CP-4 & 3.5 & 24.653 \\
CP-5 & 4.5 & 25.127 \\
CP-6 & 5.5 & 25.437 \\
\hline
\end{tabular}

\section{Conclusion}

The improved PANI/CNT composite film has been successfully polymerized by cyclic voltammetry method in the solution consisting of $0.25 \mathrm{~mol} \cdot \mathrm{L}^{-1}$ aniline monomer, $0.6 \mathrm{~mol} \cdot \mathrm{L}^{-1} \mathrm{DBSA}, 5$ $\mathrm{mol} \cdot \mathrm{L}^{-1} \mathrm{C}_{2} \mathrm{H}_{5} \mathrm{OH}$ and different concentrations of CNTs. CNTs may act as a skeleton on which aniline can polymerize in the polymerization of PAN/CNT composite film. The film exhibits a porous network structure which can offer more approaches for electron transport and facilitate ion transmission. The CNT concentration takes great effects on the performance of PAN/CNT composite film, and the electric conductivity of the film improves with the CNT concentration rising within some limits. The CNT percolation threshold of the film is about $3.5 \mathrm{wt} . \%$. The PAN/CNT film polymerized at the CNT concentration of $2.5 \mathrm{wt} . \%$, shows a reflectance change range of $38.8 \%$ at $540 \mathrm{~nm}$ as well as a coloration efficiency of $386.4 \mathrm{~cm}^{-2} / \mathrm{C}$, which means that the PAN/CNT composite film has good behavior of voltage response.

From what has been stated above, this work brings supporting evidence for the efficient effect of CNTs in obtaining composite films. The finely composite films paved the way towards novel electrochromic materials.

\section{References}

[1] Palaniappan S, John A. Prog Polym Sci, 2008, 33:732-58.

[2] Ji Yu xing, Qin Chanli, Niu Haijun, et al. Dyes And Pigments, 2015, 117:72-82.

[3] Fu Xing Qi, Jia Chun Yang, Wan Zhong Quan, et al. Organic Electronic, 2014, 11: 2702-2709.

[4] Erro, E.M., Baruzzi Ana M. Iglesias Rodrigo a. POLYMER, 2014, 10:2440-2444. 
[5] A. Reyhani, S. Z. Mortazavi, A. Z. Moshfegh, et al. Phys. Chem., 2011; 115: 6994-7001.

[6] A. Reyhani, S. Z. Mortazavi, A. Z. Moshfegh, et al. Hydrogen Energy,2011,35:231-237.

[7] Jin C, Nagaiah TC, Xia W, Spliethoff B, et al. Naoscale 2010,2:981-7.

[8] Do Nascimeto GM, Silva TB, et al. J Raman Spectro 2011, 41:1587-93.

[9] Zuzana M, Miroslava T, et al. Polymer Degradation and Stability 2012,97:1405-14.

[10] Yi Zhou, Zong-Yi Qin, et al. Electrochimca Acta 2010, 55:3904-3908.

[11] Marcela A. Bavio, Gerardo G. Acosta, Teresita Kessler. Journal of Power Sources, 2014, 245:475-481.

[12] Jianguo Deng, Xiaobin Ding, Wenchuan Zhang, et al. European Polymer Journal, 2002,38: 2497-2501.

[13] T.A. Wu, E. C. Chen, Journal of Polymer Science and Polymer Physics, 2006, 44:598-603.

[14] P.C. Ramamurthy, W.R. Harrell, R.V. Gregory, et al. Electrochem. Soc., 2007;154: 495-499.

[15] Chen Zhong Ping, Chu Dao Bai, et al. Polymer, 2007, 6:536-540.

[16] E. Lafuente, M.A. Callejas, R. Sainz, et al. Carbon, 2008,46:1909-1917.

[17] Sun Y, Wilson S R, Schuster D.I. Chem Phys Lett, 2005, 406:222 227.

[18] Wang Z, Yuan J, Han D, et al. Cryst Growth Des, 2008, 8:1827 1832.

[19] Chuang F., Yang S. Synth. Met., 2005, 150:271 277.

[20] Nagaraja M., Pattar J., Shashank N., Synth. Met. 2005, 38:271 277.

[21] Kulszewicz Bajer, Sobczak J., Hasik M. et al. Polymer, 1996, 37:25 30.

[22] Vahid Mottaghitalab, Geoffrey M. Spimks, Gordon G. Wallace. Synthetic Metals, 2005, 152: 77-80.

[23] E.N. Konyushenko, J. Stejskal, et al. Polymer, 2006, 47:5715-23.

[24] T.H. Lin, K.C. Ho. Solar Energy Mater Solar Cells, 2009, 90:506-20. 